\title{
Ole Christensen
}

\section{Functions, Spaces, and Expansions}

Mathematical Tools in Physics and Engineering

Birkhäuser

Boston $\bullet$ Basel $\bullet$ Berlin 


\section{Contents}

ANHA Series Preface v v

Preface xiii

Prologue xvii

1 Mathematical Background 1

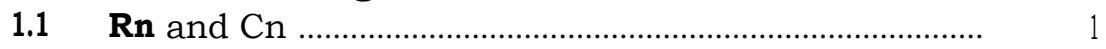

1.2 Abstract vector spaces ………………………………….........

1.3 Finite-dimensional vector spaces ..........................................

$\begin{array}{lll}1.4 & \text { Topology in } \mathrm{R}^{\mathrm{n}} & 10\end{array}$

1.5 Supremum and infimum .................................................. 11

1.6 Continuity of functions on R ......................................... 15

$1.7 \quad$ Integration and summation ……………………................ 18

1.8 Some special functions ………………………………......... 20

1.9 A useful technique: proof by induction …….................... 22

1.10 Exercises ........................................................................... 23

2 Normed Vector Spaces $\quad 29$

$2.1 \quad$ Normed vector spaces ............................................................ 29

2.2 Topology in normed vector spaces …………………........... 33

2.3 Approximation in normed vector spaces ………................ 35

2.4 Linear operators on normed spaces …………….............. 37

2.5 Series in normed vector spaces ………………………........ 40

$2.6 \quad$ Exercises ........................................................................... 
3 Banach Spaces

$\begin{array}{lll}3.1 & \text { Banach spaces } & 47\end{array}$

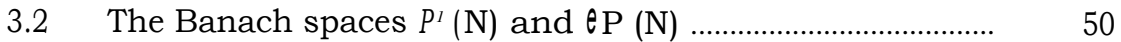

3.3 Linear operators on Banach spaces ...................................... 54

3.4 Exercises .............................................................................. 56

4 Hilbert Spaces $\quad 61$

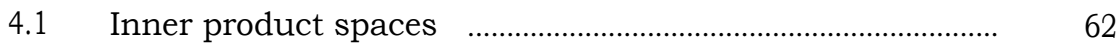

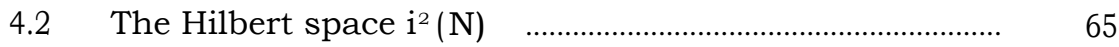

4.3 Orthogonality and direct sum decomposition ...................... 66

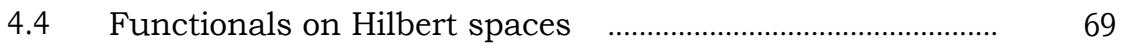

4.5 Linear operators on Hilbert spaces ............................................ 71

4.6 Bessel sequences in Hilbert spaces ............................................ 75

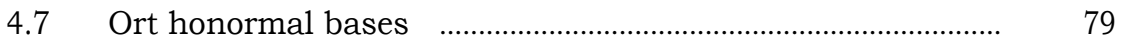

4.8 Frames in Hilbert spaces .......................................................... 84

$4.9 \quad$ Exercises ............................................................................ 85

5 The LP-spaces $\quad 93$

$5.1 \quad$ Vector spaces consisting of continuous functions ............. 94

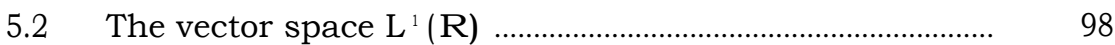

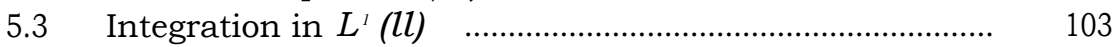

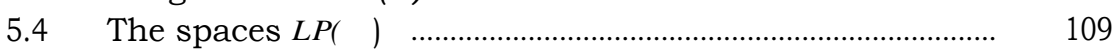

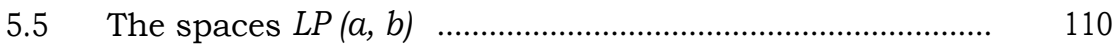

5.6 Exercises ...................................................................... 111

6 The Hilbert Space $L^{2} \quad 117$

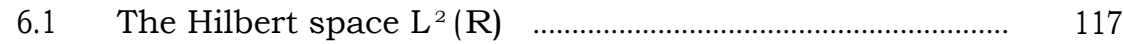

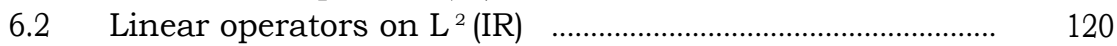

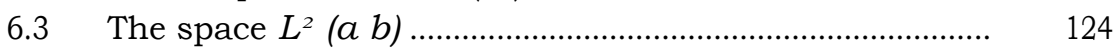

6.4 Fourier series revisited ………................................................ 126

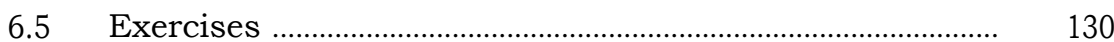

7 The Fourier Transform $\quad 135$

7.1 The Fourier transform on $\mathrm{L}^{1}$ (IR) ............................................. 135

7.2 The Fourier transform on $\mathrm{L}^{2}(\mathrm{R})$.............................................. 142

7.3 Convolution .............................................................................. 145

7.4 The sampling theorem ……..................................................... 149

7.5 The discrete Fourier transform …............................................. 154

7.6 Exercises ............................................................................ 156

8 An Introduction to Wavelet Analysis $\quad \mathbf{1 5 9}$

$8.1 \quad$ Wavelets ............................................................................. 160

8.2 Multiresolution analysis ......................................................... 162

8.3 Vanishing moments and the Daubechies' wavelets 168

8.4 Wavelets and signal processing .............................................. 174

$8.5 \quad$ Exercises ............................................................................... 176 
9 A Closer Look at Multiresolution Analysis $\quad 181$

9.1 Basic properties of multiresolution analysis $\quad$.........181

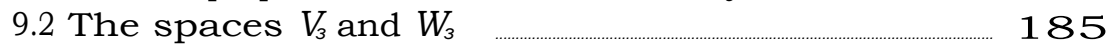

9.3 Proof of Theorem $827 \quad$........196

9.4 Proof of Theorem 8.2.11 ….....197

9.5 Exercises …....200

10 B-splines $\quad 203$

10.1 The B-splines 204

10.2 The centered B-splines $\boldsymbol{B}$, $\quad$.......208

10.3 B-splines and wavelet expansions $\quad$........209

10.4 Frames generated by B-splines $\quad$........ 210

10.5 Exercises $\quad$........ 212

11 Special Functions $\quad \mathbf{2 1 5}$

11.1 Regular St urm-Liouville problems $\quad$....... 216

11.2 Legendre polynomials $\quad$........ 222

11.3 Laguerre polynomials $\quad$....... 228

11.4 Hermite polynomials $\quad$....... 230

11.5 Exercises $\quad$........ 232

$\begin{array}{lr}\text { Appendix A } & \mathbf{2 3 9}\end{array}$

A.1 Proof of Weierstrass' theorem, Theorem 2.3.4 239

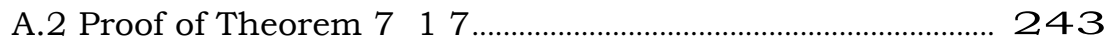

A.3 Proof of Theorem 1015 ............................................................... 246

A.4 Proof of Theorem $1122 \ldots \ldots$

$\begin{array}{ll}\text { Appendix B } & \mathbf{2 5 3}\end{array}$

B.1 List of vector spaces $\quad$....... 253

B.2 List of special polynomials $\quad$....... 255

$\begin{array}{lr}\text { List of Symbols } & 257\end{array}$

$\begin{array}{lr}\text { References } & 259\end{array}$

$\begin{array}{ll}\text { Index } & 261\end{array}$ 